\title{
Revisión de la industrialización de la vivienda y de tres experiencias de sistemas constructivos en Latinoamérica
}

\author{
Review of the industrialization of housing and of three experiences \\ of building systems in Latin America
}

Augusto Samaniego Universidad de Cuenca

\section{Recibido:}

15 de febrero 2012

Aceptado:

15 de Marzo 2012
Resumen

Este artículo trata de dar a conocer, de modo sintetizado, un conjunto de variables obtenidas de un inventario de 123 técnicas constructivas industrializadas, preparado por un grupo de investigadores latinoamericanos, recogidos en el Primer catálogo de técnicas industrializadas para la vivienda de bajo costo en América Latina y el Caribe con la coordinación del arquitecto Walter Kruk.

Las variables se refieren a la amplitud del uso de las técnicas industrializadas, el tipo de técnicas, los materiales, los periodos de desarrollo, la intensidad de la inversión y el tipo de mano de obra; las que, en general, permiten identificar las características de las empresas constructoras que han optado por la vía de la industrialización. Esta parte concluye con una descripción del perfil tecnológico de una empresa latinoamericana.

Finalmente, para ilustrar las características principales de los sistemas constructivos que utilizan técnicas industrializadas en Latinoamérica se concluye con una breve revisión de tres experiencias exitosas e innovadoras para viviendas de bajo costo como los sistemas BENO en Argentina, SANDINO en Cuba y SANCOCHO en Venezuela.

Palabras clave: Revisión, experiencias, técnicas industrializadas, vivienda, Latinoamérica.

\section{Abstract}

This article tries to present, in a synthesized way, a set of variables obtained from an inventory of 123 construction industrialized techniques, prepared by a group of American researchers, listed in the First catalog of industrialized techniques for the low-cost housing in Latin America and the Caribbean with the coordination of the architect Walter Kruk.

The variables refer to the extent of the use of industrialized techniques, the kind of techniques, materials, periods of development, the intensity of the investment and the type of labor; which, in general, allow to identify the characteristics of the construction companies that have opted for the path of industrialization. This part concludes with a description of the technological profile of a Latin American company.

Finally, to illustrate the main features of the building systems that use industrialized techniques in Latin America it concludes with a brief review of three successful and innovative experiences for low cost housing as the sytems BENO in Argentina, SANDINO in Cuba and SANCOCHO in Venezuela.

Keywords: Review experiences, industrialized techniques, housing, Latin America. 


\section{Introducción:}

La construcción es una las fuentes principales de empleo en el país, la modalidades generalizadas son: el trabajo temporal, la subcontratación o a destajo. Los trabajadores de la construcción están sujetos a la inestabilidad laboral y a mayores accidentes en su trabajo con relación a otras actividades industriales. La mano de obra empleada en la construcción tiene un bajo nivel de formación, lo que impide mejorar la calidad y la productividad. En la construcción, la mano de obra sin ninguna preparacióninterviene en un porcentaje significativo, recurriendo en numerosos casos sólo al uso del esfuerzo físico.

A la investigación tecnológica en la cons trucción no se le da la debida importancia en las políticas de las instituciones que se dedican a la investigación y por lo tanto cuenta con muy poco apoyo financiero. Las empresas constructoras por su debilidad inversora o por falta de visión no están por lo general en capacidad de incorporar I+D (Investigación y Desarrollo). En las universidades y escuelas politécnicas no se presta la atención debida a la formación científica y técnica en el área de los métodos y procesos de producción de la construcción.

La debilidad en la investigación, la falta de formación técnica de profesionales y la escasa capacitación de la mano de obra son factores que impiden incorporar los adelantos científicos y las técnicas modernas a la construcción.

De continuar las condiciones actuales de producción primordialmente artesanal y de escaso desarrollo tecnológico imperante en el país, resultaría imposible en la actualidad y a futuro que el sector de la construcción esté en capacidad de atender la necesidad de construir masivamente viviendas de calidad frente al creciente déficit habitacional. Esta situación crítica en cuanto al déficit habitacional y la debilidad productiva de la construcción es generalizable a los países de América Latina y el Caribe.
En América Latina existen muy pocos estudios que se dediquen al tema de la industrialización en la construcción de vivienda. Julián Salas, en su significativa obra América Latina: una industrialización posible, realiza un análisis crítico sobre la situación de la industrialización en Europa, luego examina detalladamente las potencialidades instaladas en la industrialización de la vivienda y propone algunos métodos que podrían servir para promover y transferir la tecnología a los países de América Latina y el Caribe.

Ante este panorama crítico y como una primera respuesta, un grupo de profesionales de América Latina y el Caribe convocados por el Programa Cooperación Iberoamericana de Ciencia y Tecnología para el Desarrollo, CYTED-D Proyecto XIV. 2: Técnicas constructivas industrializadas para viviendas de bajo coste ${ }^{1}$, como parte del proyecto realizó un inventario de los sistemas constructivos industrializados y llegó a la conclusión de que para enfrentar los grandes volúmenes de obra por construir, la vía apropiada es el apoyo y fomento de los procesos y técnicas industrializadas cimentadas en la realidad y condiciones de cada país.

Una de las metas del inventario fue la publicación en 1992 del Catalogo iberoamericano de técnicas constructivas industrializadas para viviendas de interés social, que sirve de base para el presente artículo.

\section{Características de la industrialización de la vivienda en Latinoamérica}

En el Catálogo iberoamericano de técnicas constructivas industrializadas para viviendas de interés social se recoge información de 123 técnicas constructivas industrializadas (con mayor o menor grado de industrialización) mediante fichas aplicadas a empresas o instituciones responsables de los sistemas o subsistemas constructivos. La información recogida es significativa, representa a once países y se estima que se han construido del orden de cuarenta millones de metros cuadrados, equivalentes a unas ochocientas mil viviendas en Latinoamérica por el sector industrializado.

Del total de las 123 técnicas industrializadas, 62 casos $(50,4 \%)$ abarcan todos o gran parte de los sectores de la obra del edificio; 40 casos $(32,5 \%)$ abarcan varios componentes funcionales (entrepisos y cubiertas o cerramientos exteriores e interiores); y 21 casos $(17 \%)$, corresponden a un solo tipo de componente (paneles livianos para cerramientos interiores o exteriores, viguetas o bodeguillas para entrepisos) 
La técnica predominante es la prefabricación con el $94 \%$, que se aplica en todo o parte del edificio, en el $29 \%$ se incorpora el molde racionalizado; y en el $15 \%$, sistemas no convencionales (morteros proyectados sobre mallas metálicas o vegetales, cerramientos livianos de varias capas, etc.). La incorporación de técnicas tradicionales tiene una importante participación en el proceso de producción con el $60 \%$ del conjunto de componentes (cimientos, paredes apiladas y cubiertas).

De los materiales utilizados en la fabricación de las distintas soluciones industrializadas, el hormigón armado es predominante con el 60\%; el acero con el $19 \%$, la albañilería con el $14 \%$, el ferrocemento con el $6 \%$ y otros materiales (fibrocemento, plásticos, aceros especiales, etc.), con el $54 \%$.

Casi la mitad del uso de las técnicas industrializadas son de reciente desarrollo, 60 casos son posteriores a 1980, 21 durante la década del setenta, 12 durante la década del sesenta y 7 anteriores a 1960 .

Para medir la utilización de la inversión se han elegido tres parámetros: el tipo de plantas de prefabricación, el tipo de equipos utilizados en montaje, y el peso de los elementos prefabricados. Se observa que el $61 \%$ de los casos se realiza en plantas fijas y en el $52 \%$ se realiza el montaje de forma manual.

En lo relativo a la utilización de la mano de obra especializada, ésta se concentra en la mayoría de los casos en la planta y la mano de obra no especializada en el montaje en la obra.

En resumen, según Julián Salas, el perfil medio de una empresa latinoamericana de industrialización de viviendas se puede definir por las siguientes características:

- La mayoría de las técnicas industrializadas son incorporadas por empresas privadas que tratan de incluir diversos tipos de demandas aparte de la vivienda y de abarcar todo el proceso (proyecto, producción, transporte, montaje, acabados y promoción).

- Las técnicas industrializadas en las empresas, en su mayoría, han sido implementadas hace menos de diez años para la construcción de viviendas, impulsadas por la realización de un gran volumen de viviendas.

- La prefabricación en hormigón armado en estructuras de cerramientos en combinación con soluciones tradicionales para cimentación y cubierta es lo predominante.Incorpora materiales o instalaciones no convencionales, combinando la prefabricación con el moldeo racionalizado en el sitio de losas $\mathrm{u}$ otros componentes de la vivienda.La característica general de la forma de producción es que la inversión es modesta en lo que se refiere a la planta fija y el montaje es manualmente o con medios auxiliares y equipos livianos de elevación. Regularmente en los procesos de producción utiliza mano de obra no especializada pero siempre en el contexto de la economía formal.

A continuación, se presentan en forma resumida, tres sistemas constructivos representativos de América Latina que utilizan técnicas industrializadas de Argentina, Cuba y Ve-

nezuela.
1.1 Sistema BENO (Argentina)

El sistema BENO fue investigado y desarrollado por CEVE, se han construido viviendas con este sistema desde hace treinta años en Argentina. Esta experiencia tecnológica se ha transferido a Brasil y Uruguay. El sistema BENO se destaca por su rapidez y bajo costo, como también por su aptitud para la auto construcción comunitaria.

CEVE es el Centro Experimental de la Vivienda Económica fundado en 1967, donde se han desarrollado diversos programas constructivos con la finalidad de obtener viviendas económicas. Este centro experimental ha usado materiales tradicionales en forma no tradicional como es el caso de estudio BENO, donde se utilizaron ladrillos comunes de tierra cocida para construir placas premoldeadas para levantar mampostería. Esta tecnología fue elaborada por los arquitectos: H. Barreta, H. Massuh, G. Bosio y D. Pipa hace treinta años en Argentina.

El sistema constructivo BENO fue concebido, por un lado, como la mejor manera de sacarle provecho al ladrillo de barro común cocido, de fabricación artesanal como elemento básico para elaborar módulos constructivos mayores mediante la prefabricación de placas de ladrillo premoldeadas que estén unidos por juntas de hormigón y, por otro, racionalizar el proceso de construcción de viviendas prefabricando partes con dimensiones estandarizadas, dando más y mejor tiempo a los procesos seriados y controlables y menos al montaje. El sistema constructivo BENO está compuesto por placas de muros, placas de techos y ventanas de hormigón, con instalación eléctrica y plancha de poliestireno expandido incorporada en placa. (Ver Gráficos N²1,23 siguiente página)

Las placas de cerámica armada se producen prefabricando en el suelo con la guía de un molde de madera o hierro en el que se colocan tres hileras de ladrillos unidas mediante junta de mortero armado. Las dimensiones habituales de la placa de cerámica armada para pared es 0,43 por 2,27 m. y para techo es de 0,43 por 0,90 m. 


\section{Traslado de paneles}

1. Parado de placas interiores con escuadra

2. Parado de placas exteriores

3. Colocación de armaduras de columnas

4. Hormigonado
A. Losa de cimentación
B. Placas de ladrillo y materiales-equipos

1. Montaje de reglas para fijación de placas

2. Parado de placas exteriores

3. Colocación de polietileno expandido

4. Parado de placas interiores

5. Parado de ventanas

La prefabricación de las placas puede realizarse con una inversión mínima en equipos y la planta de producción puede funcionar al aire libre. Para la prefabricación se requiere contar básicamente con moldes que pueden ser de madera o hierro, equipos livianos (hormigonera), herramientas (carretillas y herramientas de albañilería) y los materiales (ladrillos, cemento, arena y varillas de hierro).

El peso de la placa de cerámica armada es de aproximadamente $60 \mathrm{~kg}$, lo que permite que dos personas la puedan transportar, sin gran esfuerzo, facilitando el montaje en la obra.

El montaje, además de rápido es sencillo, y puede realizarse tras un mínimo proceso de capacitación. La secuencia del montaje comienza con la izada manual de las placas de cerámica en las esquinas de lo que conformarán las paredes con el apoyo de puntales, luego con la izada manual de las placas de cerámica que conforman las paredes en las que se incluyen los marcos de ventanas en hormigón, después con el hormigonado de vinculación, cadena superior y juntas verticales, posteriormente la colocación de las placas cerámicas de cubierta y finalmente las instalaciones sanitarias, eléctricas, y acabados.

Las ataduras entre placas y los encadenados que las solidifican propende que se comporte con una pieza estructural única.

La modulación flexible del sistema constructivo permite versatilidad en el diseño arquitectónico. Tanto las instalaciones como los acabados admiten numerosas variantes en función de los requerimientos del usuario.

1. Mojado de placas previo hormigonado de nervios

2. Colado de juntas verticales y juntas de carpintería

3. Tomado de juntas verticales

4. Colocación de armadura longitudinal superior

5. Colocación de caño flexible electricidad

6. Colado de nervio longitudinal

7. Aplicación de mortero
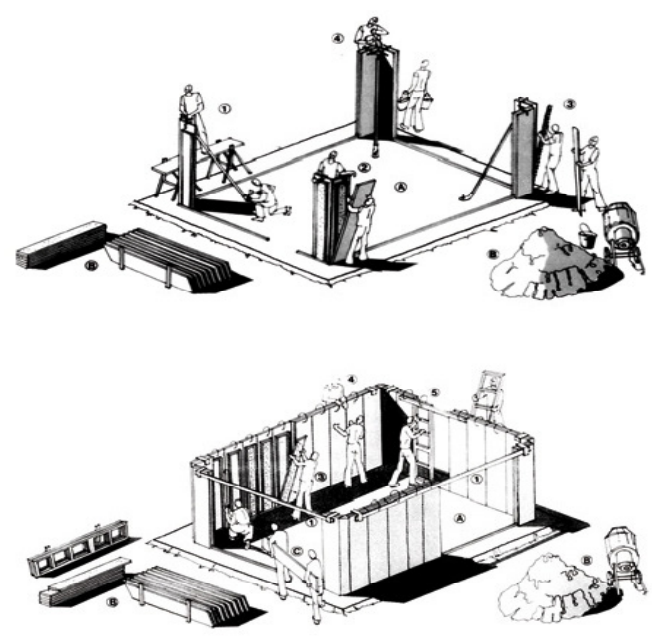

Gráfico № 1,2

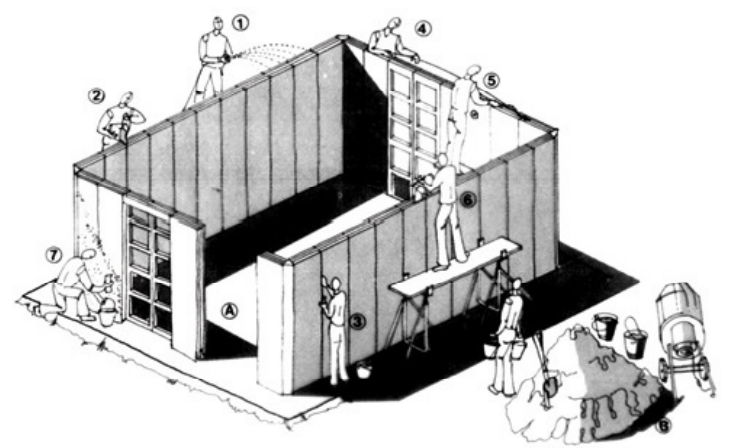

Gráfico № 3
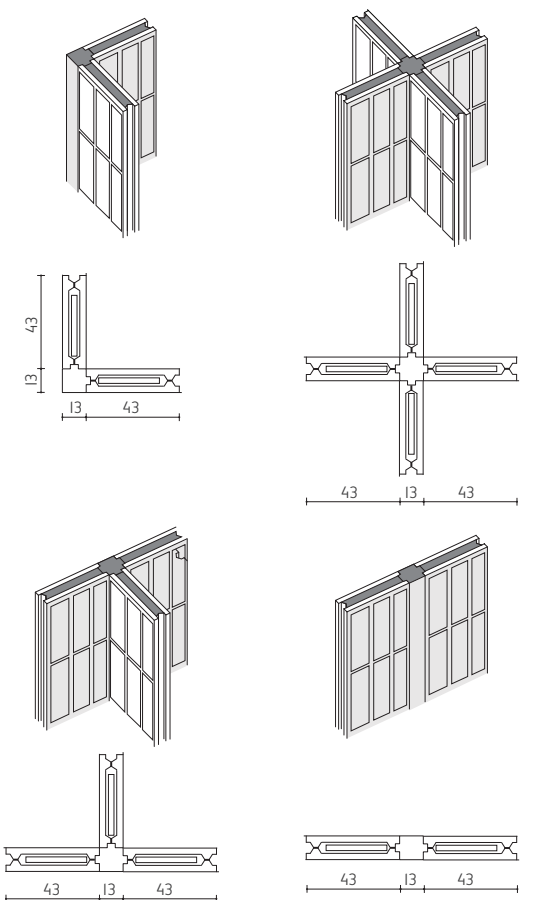
Revisión de la industrialización de la vivienda y de tres experiencias...

\subsection{Sistema SANDINO (Cuba) ${ }^{3}$}

El Sistema SANDINO, de origen cubano, es uno de los más utilizados en Latinoamérica para la vivienda de una o dos plantas. Según Julián Salas, el sistema de producción SANDINO está en el punto intermedio entre las plantas de producción de grandes paneles y los gérmenes productivos, mucho más cercano de la industrialización posible en Latinoamérica.

El sistema de elementos prefabricados SANDINO ofrece las ventajas de la fabricación a bajo costo, rapidez de ejecución y flexibilidad de diseño. El sistema originalmente concebido para viviendas se ha ampliado a la aplicación de la construcción de escuelas, puestos de salud, albergues, oficinas, etcétera.

Es un sistema que se adapta fácilmente a las necesidades cambiantes de la construcción mediante formas de prefabricación con diferentes tecnologías de producción. No requiere mano de obra especializada y es posible realizar el producto mediante el uso de tecnologías manu

El sistema se basa en paredes compuestas por pequeñas columnas y paneles prefabricados de hormigón. La modulación es de 1040 mm entre ejes de columnas y el espacio entre ellos es ocupado por 5 paneles de hormigón o ventanas.

La luz para vivienda está entre los 3,12 m o 4,16 m; en el caso de aulas, naves y otras obras puede alcanzar hasta los 6,24 m, mediante vigas o cerchas espaciadas a $3,12 \mathrm{~m}$, con una columna especial en estos puntos. Las columnas son de hormigón ligeramente armado tiene una sección de 110 por $110 \mathrm{~mm}$, una altura de 2435 $\mathrm{mm}$, y su peso oscila entre los 63 y $71 \mathrm{~kg}$ dependiendo del tipo de sección.

El tipo de sección de columnas está en función de la sección básica y las variantes son producto de las pestañas que sirven de alojamiento a los paneles de pared por una o varias caras, dando opción a utilizar diferentes posiciones de la columna dentro del diseño de la vivienda.

Los paneles de pared son de hormigón siempre sin refuerzo de acero, poseen dimensiones de 945 por 468 por $60 \mathrm{~mm}$ y pesa $65 \mathrm{~kg}$. Para los paneles pueden utilizarse materiales como cerámica, cáscara de arroz con cemento, hormigón con fibras vegetales y otros. (Ver Gráficos $\mathrm{N}^{\circ} 4,5$ )

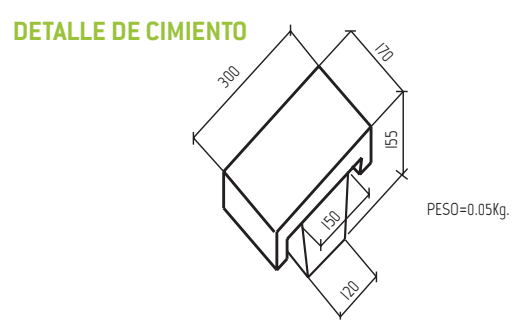

DETALLE DE ARMADO DE PARED

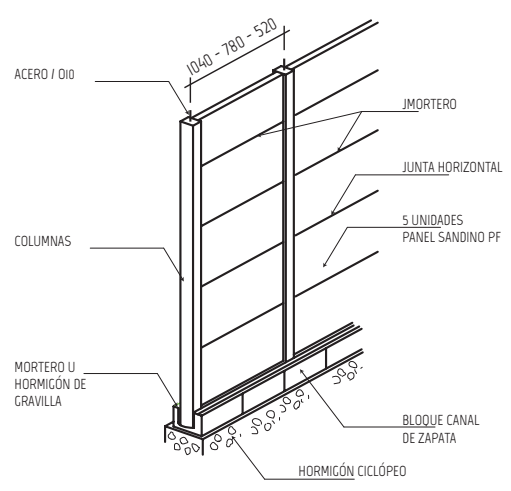

SECCIONES CONSTRUCTIVAS

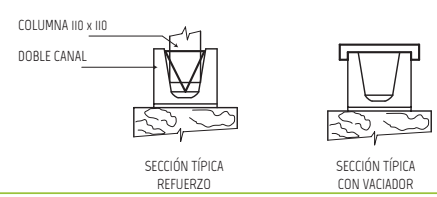

Gráfico № 4

TIPOS DE PANELES
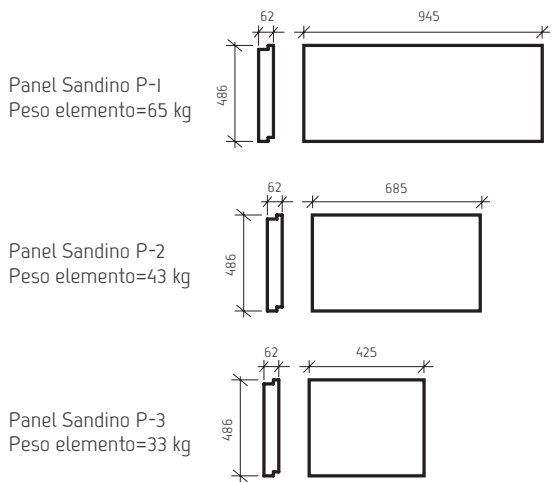

TIPOS DE COLUMNAS

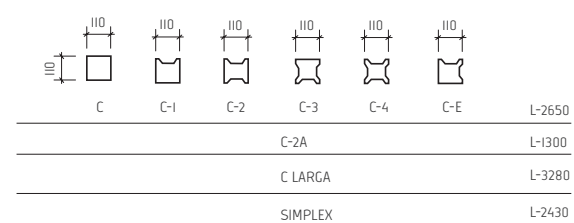

Gráfico № 5 


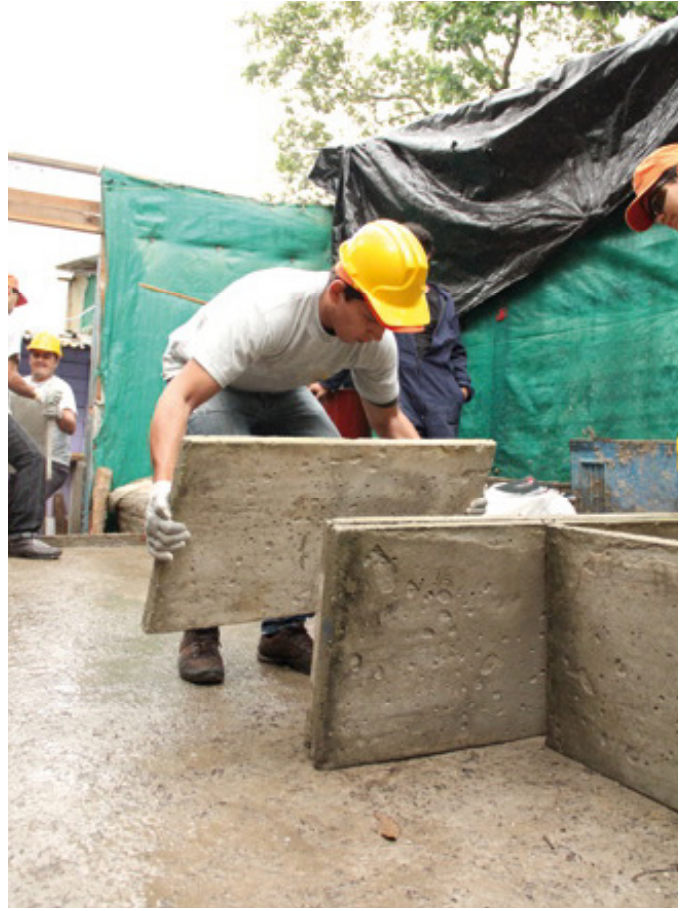

\subsection{Sistema SANCOCHO (Venezuela) ${ }^{4}$}

El sistema venezolano SANCOCHO utiliza técnicas industrializadas de construcción, que pueden ser aplicadas por el sector formal de la construcción o por los propios usuarios mediante la autoconstrucción.

El sistema SANCOCHO está basado en elementos livianos de manejo manual, producidos con perfiles de chapas dobladas y concreto. El acero y el cemento son de producción venezolana abundante y a precios competitivos.

El sistema constructivo SANCOCHO está compuesto por los siguientes elementos:

- Vigas de cimiento: elementos prefabricados lineales o en forma de " $T$ " $\mathrm{O}$ " $\mathrm{L}$ " reforzados con barras de acero y de sección transversal troncocónica de $15 \mathrm{~cm}$ de altura, la base es de $20 \mathrm{~cm}$ y el tope es de $15 \mathrm{~cm}$.

- Columnas: compuestas por pletinas y perfiles de lámina doblada con una sección nominal en planta de $6 \mathrm{~cm}$ por $3,5 \mathrm{~cm}$, las cuales permiten, según su tipo, la llegada de una, dos, tres, o cuatro paredes. Las columnas son de altura variable desde $2,40 \mathrm{~m}$ hasta $3,90 \mathrm{~m}$.

- Paredes, vigas y losas: son elementos de $3 \mathrm{~cm}$ de espesor, se construyen mediante un bastidor

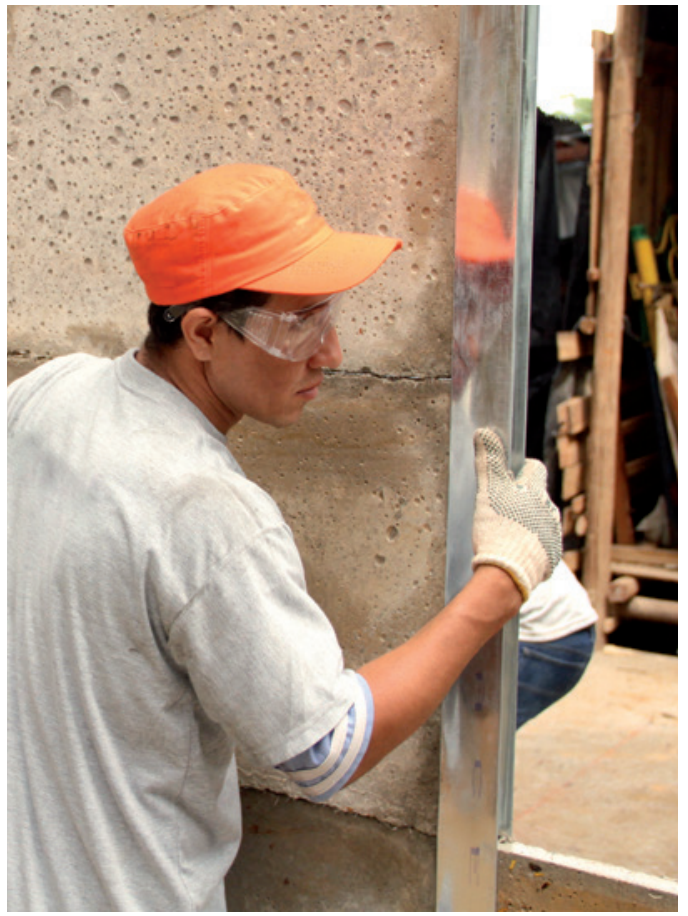

conformado por perfiles de chapa doblada de 1,1 $\mathrm{mm}$ de espesor y rigidizada mediante varilla de hierro de $5 \mathrm{~mm}$ de diámetro colocados diagonalmente. Estos bastidores además de servir de encofrados laterales, sirven de armadura que se integra con el concreto conformando el elemento. El elemento delgado de acero-concreto está diseñado para resistir las tensiones a las que van a estar sometidos durante el transporte, montaje y a las acciones permanentes en su uso.

El sistema constructivo SANCACHO está organizado para permitir una conformación espacial a base de una retícula ortogonal en planta de 90 × $90 \mathrm{~cm}$ y verticalmente, en módulos de 30, 45, 60 y $90 \mathrm{~cm}$. Las alturas libres de entrepiso son habitualmente de 2,40 $\mathrm{cm}$ pero se puede llegar a alturas de $3,90 \mathrm{~m}$. Las luces libres en planta en una dirección pueden ser desde $1,80 \mathrm{~m}$ hasta $3,60 \mathrm{~m}$ para viviendas. Para otro tipo de edificaciones puede tener una luz de 7,20 m con los debidos refuerzos.

\section{A manera de conclusión}

La difusión de las experiencias industrializadas y sus características en el uso de tecnologías apropiadas, especialmente las que se vienen realizando en Latinoamérica son el primer paso para demostrar que existen potencialidades desconocidas en nuestro continente y que pueden ser aprovechadas por los investigadores, profesores, profesionales, estudiantes y técnicos, para motivar la innovación y el desarrollo científico del sector de la construcción tecnológicamente atrasado en los métodos y procesos de producción masiva de viviendas en el país. 
Revisión de la industrialización de la vivienda y de tres experiencias...

\section{Bibliografía}

- Kruk, Walter, Coordinador CYTED Proyecto XIV. 2 en Uruguay. Anales del II Curso iberoamericano de técnicas constructivas industrializadas para viviendas de interés social. Conjunto demostrativo de tecnologías $\vee$ Centenario. Descripción de sistemas constructivos aplicados. Tomo 3. Empresa CEVE. Característica del Sistema BENO Montevideo, Uruguay. Noviembre 1993.

- Peña, José A. Jefe del Proyecto. CYTED Proyecto XIV.2. Técnicas constructivas industrializadas para viviendas de interés social. Curso teóricopráctico, Puerto Ordaz (Venezuela). Tomo III Monografías de las tecnologías participantes. Sistema Sancocho-Venezuela. Puerto OrdazVenezuela. 14 octubre-22 noviembre, 1991.

- Salas, Julián. La industrialización posible de la vivienda latinoamericana. ESCALA. Tecnologías para viviendas de interés social. Bogotá. Colombia. 2000.

\section{Créditos de fotografías y gráficos}

- Fotografías No. 1, 2 y 3 Documento PDF Sistema BENO

- Gráficos No. 1, 2, 3 y 4 Documento PDF Sistema BENO

- Fotografía No. 4 Documento CTDMC PDF Sistema Sandino

- Fotografías No. 5 y 6 Documento PDF Sistema Sandino

- Gráficos No. 5, 6, 7, 8 y 9Documento PDF Sistema Sandino

- Gráfico No. 10 Catálogo iberoamericano de técnicas constructivas industrializadas para viviendas de interés social. Proyecto CYTED 1993.

- Gráfico No. 12 Documento WORD (internet), Análisis sistema Sancocho, Ing. José Peña U. de la oficina técnica OTIP.C.A. Caracas-Venezuela.

- Fotografías No. 7, 8, 9 y 10 Documento PDF (internet) Ing. José Adolfo Peña U. 